\title{
Inhibition of LHX2 by miR-124 suppresses cellular migration and invasion in non-small cell lung cancer
}

\author{
QINGHUI YANG ${ }^{1,2^{*}}$, LIANG WAN $^{1,2^{*}}$, CAN XIAO $^{3 *}$, HAIBO HU $^{4 *}$, LONGQIANG WANG $^{1,2}$, \\ JUN ZHAO ${ }^{2,5}$, ZHE LEI $^{1,2}$ and HONG-TAO ZHANG ${ }^{1,2}$
}

\begin{abstract}
${ }^{1}$ Soochow University Laboratory of Cancer Molecular Genetics, Medical College of Soochow University; ${ }^{2}$ Suzhou Key Laboratory for Molecular Cancer Genetics, Suzhou, Jiangsu 215123; ${ }^{3}$ Department of Stomatology, The First Affiliated Hospital of Soochow University, Medical College of Soochow University, Suzhou, Jiangsu 215006; ${ }^{4}$ Department of Cardiothoracic Surgery, Huai'an Second People's Hospital, The Affiliated Huai'an Hospital of Xuzhou Medical University, Huai'an, Jiangsu 223200; ${ }^{5}$ Department of Thoracic and Cardiovascular Surgery, The First Affiliated Hospital of Soochow University, Medical College of Soochow University, Suzhou, Jiangsu 215006, P.R. China
\end{abstract}

Received December 5, 2015; Accepted May 11, 2017

DOI: $10.3892 / 01.2017 .6607$

\begin{abstract}
Downregulated microRNA (miR)-124 is common in numerous types of cancer, including non-small cell lung cancer (NSCLC). A previous study by the authors demonstrated that LIM-homeobox domain 2 (LHX2) was upregulated and promoted cell growth in NSCLC. However, whether LHX2 affects the migratory and invasive abilities of NSCLC cells and the association of LHX2 with miR-124 remains unclear. The present study revealed that miR-124 expression was frequently decreased in human NSCLC cells and tissues and negatively correlated with LHX2 expression, which was increased in NSCLC cells and tissues. Furthermore, the transfection of miR-124 mimic significantly inhibited endogenous expression of LHX2 mRNA and protein in A549 and H1299 cells, and miR-124 inhibitor promoted LHX2 expression. Of note, overexpression of miR-124 in A549 and H1299 cells attenuated cellular migratory and invasive abilities, and this was observed in LHX2-silenced A549 and H1299 cells. Knockdown of miR-124 augmented the migratory and invasive abilities in A549 and H1299 cells. The 3'-untranslated region of LHX2 transcript has also been identified to be a putative target of miR-124. Taken together, the results revealed that miR-124 may inhibit migration and invasion by repressing
\end{abstract}

Correspondence to: Dr Hong-Tao Zhang or Dr Zhe Lei, Soochow University Laboratory of Cancer Molecular Genetics, Medical College of Soochow University, 199 Ren'ai Road, Suzhou, Jiangsu 215123, P.R. China

E-mail: htzhang@suda.edu.cn

E-mail: leizhe@suda.edu.cn

*Contributed equally

Key words: non-small cell lung cancer, microRNA-124, LIM-homeobox 2, migration, invasion
LHX2 expression in NSCLC cells. The findings of the present study suggested that overexpression of miR-124 or silencing of LHX2 may provide a therapeutic strategy for advanced NSCLC.

\section{Introduction}

Lung cancer is the leading cause of cancer-associated mortality worldwide. Non-small cell lung cancer (NSCLC) accounts for $\sim 85 \%$ of all lung cancer cases (1). The prognosis for patients with NSCLC remains poor, with a 5-year survival rate of $\sim 17 \%$ (2). The low survival rate of NSCLC is primarily due to tumor cell metastasis (3). Therefore, it is necessary to investigate the mechanisms underlying NSCLC metastasis.

MicroRNAs (miRNAs) are 19-24 nucleotides small non-coding RNAs, which directly bind to target motifs in mRNAs and post-transcriptionally suppress gene expression by transcript degradation or translational repression $(4,5)$. There is growing evidence that miRNA dysfunction is involved in the growth and/or metastasis of various types of human tumors (6,7). MicroRNA (miR)-124, a widely studied miRNA, was demonstrated to be downregulated and regarded as a tumor suppressor in breast, gastric and bladder cancer, as well as head and neck squamous cell carcinoma (8-11). $\mathrm{Xi}$ et al (12) and Sun et al (13) recently demonstrated that miR-124 significantly repressed cell invasion and metastasis in colorectal cancer and NSCLC. Decreased expression of miR-124 was associated with poor prognosis in patients with breast cancer or NSCLC $(14,15)$. These results suggested that miR-124 may serve an important role in the regulation of tumor metastasis. Although miR-124 may inhibit NSCLC metastasis by targeting MYO10 (13), the other targets of miR-124 in this process cannot be excluded.

LIM-homeobox domain 2 (LHX2), a member of the LIM-homeodomain proteins, was previously reported to serve an important role in the control of lymphoid and neural cell differentiation and brain and eye development (16). LHX2 was also implicated in the development of various 
types of human tumors. For example, LHX2 may promote breast cancer cell growth and metastasis by stimulating the activity of platelet-derived growth factor subunit B signaling pathway (17). The authors of the present study previously demonstrated that LHX2 was highly expressed and may serve an oncogenic role in NSCLC (18). Although data of the previous study demonstrated that knockdown of LHX2 inhibited NSCLC cell proliferation and arrested cell cycle at $\mathrm{G}_{1}$ phase (18), it remains unclear whether LHX2 affects the migratory and invasive abilities of NSCLC cells.

Low miR-124and high LHX2 expression levels have been observed in different cancer types in humans. Therefore, there may be a link between miR-124 and LHX2 in NSCLC. In order to investigate this hypothesis, the present study first used TargetScanHuman v7.0 software to predict miRNA targets and demonstrated that the 3'-untranslated region (3'-UTR) of the LHX2 transcript was a putative target of miR-124. therefore, this attracted our attention to the association between miR-124 and LHX2 in NSCLC.

To the best of our knowledge, the present study is the first time that the role of LHX2 in NSCLC cell invasion and an association between miR-124 and LHX2 in NSCLC has been investigated. The results revealed that LHX2 has an important role in promoting NSCLC cell migration and invasion, which maybe controlled at least partially by miR-124.

\section{Materials and methods}

Cell culture. Human bronchial epithelial (HBE) cells (Bogoo Biotechnology, Shanghai, China) and human NSCLC cells A549, LTEP-a2, H1299 (two lung adenocarcinoma cell lines), H226 (lung squamous carcinoma cell line), 95C and 95D (two giant-cell carcinoma cell lines) and H460 (large cell carcinoma cell line) from the Cell Bank of the Chinese Academy of Sciences (Shanghai, China), were cultured in RPMI-1640 medium (HyClone, Logan, UT, USA) supplemented with $10 \%$ fetal bovine serum (FBS; Invitrogen; Thermo Fisher Scientific, Inc., Waltham, MA, USA), L-glutamine and $50 \mathrm{U} / \mathrm{ml}$ each of penicillin and streptomycin (Invitrogen; Thermo Fisher Scientific, Inc.) at $37^{\circ} \mathrm{C}$ with $5 \% \mathrm{CO}_{2}$ in a humidified atmosphere.

Tissue samples. A total of 40 paired tumor tissues and adjacent noncancerous tissues were collected, by surgical resection, from patients with NSCLC at the First Affiliated Hospital of Soochow University (Suzhou, China) between April 2007 and December 2013. The demographic and clinical features were described in Table II. Written informed consent was obtained from all patients prior to enrollment in the present study. Histological and pathological diagnostics for patients with NSCLC were evaluated according to the Revised International System for Staging Lung Cancer (19). None of the patients received chemotherapy or radiotherapy prior to tissue sampling. The samples were snap-frozen in liquid nitrogen and stored at $-80^{\circ} \mathrm{C}$. The present study was approved by the Academic Advisory Board of Soochow University.

RNA extraction, cDNA synthesis and reverse transcription-quantitative polymerase chain reaction ( $R T$-qPCR). Total RNA was isolated from NSCLC cells and human NSCLC tissues using the HP Total RNA kit (Omega Bio-Tek,
Inc., Norcross, GA, USA). Purified RNA was reversed transcribed to cDNA using the M-MLV cDNA Reverse Transcription kit (Invitrogen; Thermo Fisher Scientific, Inc.), and the sequences of the primers used are presented in Table I. RT-PCR was performed using a Platinum SYBR Green qPCR SuperMix-UDG kit (Thermo Fisher Scientific, Inc.), according to the manufacturer's protocol on Roche Lightcycler 96 (Roche Diagnostics, Basel, Switzerland). The thermocycling conditions for qPCR were as follows: $95^{\circ} \mathrm{C}$ for $5 \mathrm{~min}, 40$ cycles of $95^{\circ} \mathrm{C}$ for $10 \mathrm{sec}, 60^{\circ} \mathrm{C}$ for $30 \mathrm{sec}$, followed by $72^{\circ} \mathrm{C}$ for $10 \mathrm{~min}$. Each RT-qPCR experiment was performed in triplicate. The relative expression levels of LHX2 mRNA and miR-124 were normalized to $\beta$-actin mRNA and U6, respectively, and evaluated according to the $2^{-\Delta \Delta \mathrm{Ct}}$ method (20).

Western blot analysis. Cells and tissues were lysed and subjected to western blot analysis as previously described (7). Antibodies applied in the analysis were as follows: rabbit anti-LHX2 (catalog no. sc-367972; dilution, 1:1,000; Santa Cruz Biotechnology, Inc., Dallas, TX, USA) and mouse anti- $\beta$-actin (catalog no. CW0096M; dilution, 1:2,000; CWBIO, Beijing, China) primary antibodies, and horseradish peroxidase (HRP)-conjugated goat anti-rabbit (catalog no. CW0103S; dilution, 1:3,000; CWBIO) or HRP-conjugated goat anti-mouse secondary antibodies (catalog no. CW0107S; dilution, 1:3,000; CWBIO). The LHX2 expression level was normalized to $\beta$-actin.

Plasmid construction and luciferase assay. A 72 base pair (bp) DNA sequence of LHX2 3'-UTR containing a predicted miR-124 target site (position 315-312, predicted using TargetScanHuman software; version 7.0; www.targetscan.org) was directly synthesized (GENEWIZ, Suzhou, China) and subcloned into a dual-luciferase report vector, psiCHECK-2 (Promega Corporation, Madison, WI, USA), in order to generate a psiCHECK-2-LHX2 3'-UTR-wildtype. In addition, another similar $72 \mathrm{bp}$ DNA fragment containing a mutant miR-124 target site was synthesized for construction of a psiCHECK-2-LHX2 3'UTR-mutant vector (GENEWIZ). Subsequently, each of the luciferase constructs was co-transfected with miR-124 mimics (5'-UAAGGCACGCGGUGA AUGCC-3') or a scrambled negative control (miR-NC; 5'-UUC UCCGAACGUGUCACGUTT-3') into A549 and H1299 cells, respectively. Following transfection for $48 \mathrm{~h}$ at $37^{\circ} \mathrm{C}$, the cells were lysed and analyzed for luciferase activities using the dual-luciferase reporter assay system (Promega Corporation). Each experiment was performed in triplicate. Results are represented as relative to Renilla luciferase activities, which were normalized to firefly luciferase activities. All transient transfections, including anti-miR-124 (5'-GGCAUUCACC GCGUGCCUUA-3') and anti-miR-NC (5'-CAGUACUUU UGUGUAGUACAA-3') were performed using Lipofectamine 2000 (Thermo Fisher Scientific, Inc.), according to the manufacturer's instructions.

Transient RNA interference. Two short interfering RNAs (siRNA), which specifically target the LHX2 transcript were directly synthesized (GenePharma Co., Ltd., Shanghai, China). Sequences for LHX2 siRNAs were as follows: si-LHX2-1, 5'-GCT TCG GACCAT GAAGTC TTA-3'; si-LHX2-2, 
Table I. Primers for reverse transcription or amplification of miR-124 and LHX2 mRNA.

\begin{tabular}{ll}
\hline Primer & \multicolumn{1}{c}{ Sequence (5'-3') } \\
\hline RT & \\
U6 & CGAGCACAGAATCGCTTCAC \\
& GAATTTGCGTGTCAT \\
miR-124 & GTCGTATCCAGTGCAGGGTCCG \\
& AGGTATTCGCACTGGATACGAC \\
& GGCATT \\
qPCR & \\
U6, forward & CGAGCACAGAATCGCTTCA \\
U6, reverse & CTCGCTTCGGCAGCACATAT \\
miR-124, forward & GTGCAGGGTCCGAGGTATT \\
miR-124, reverse & GCTAATAAGGCACGCGGTG \\
$\beta$-actin, forward & CACAGAGCCTCGCCTTTGCC; \\
$\beta$-actin, reverse & ACCCATGCCCACCATCACG \\
LHX2, forward & TTCCAGAACGCCCGAGCCAA \\
LHX2, reverse & GGGGCTAGTCAAGTCTGTC \\
\hline
\end{tabular}

miR, microRNA; LHX2, LIM-homeobox domain 2; RT-PCR, reverse transcription polymerase chain reaction; qPCR, quantitative PCR.

5'-GCA ACCTCT TACGGCAGGAAA-3'. A scrambled sequence (5'-TTCTCCGAACGTGTCACGT-3') was used as the negative control (si-NC). The cells were transiently transfected with 100 pmol of siRNA or si-NC for $48 \mathrm{~h}$ at $37^{\circ} \mathrm{C}$, using Lipofectamine 2000 (Thermo Fisher Scientific, Inc.). Following 3 days, the cells were collected for further experiments.

Transwell migration and Matrigel invasion assays. Transwell migration and Matrigel invasion assays were performed using Transwell plates with $0.8 \mu \mathrm{m}$ pore polycarbonate membranes (BD Biosciences, Franklin Lakes, NJ, USA). A549 and H1299 cells $\left(5 \times 10^{4}\right)$ supplemented with $1 \%$ FBS were seeded in the upper chamber [for the invasion assay, Matrigel (Corning, NY, USA) was added in the upper chamber prior to cell seeding] and allowed to invade to the reverse side of the chamber under chemoattractant conditions with $10 \%$ FBS medium in the lower chamber. Following incubation for $48 \mathrm{~h}$ at $37^{\circ} \mathrm{C}$, the cells on the upper side were wiped, and invaded cells on the lower side were stained with $1 \%$ crystal violet (BioTime Inc., Alameda, CA, USA) and imaged and counted under three microscopic fields (light microscope; magnification, x100).

Statistical analysis. All statistical analyses were performed using GraphPad Prism version 5.02 software (GraphPad Software, Inc., La Jolla, CA, USA). Differences in LHX2 and miR-124 expression levels between NSCLC tissues (T) and adjacent noncancerous lung tissues( $\mathrm{N})$ were analyzed using a paired t-test (two-tailed), and data are presented as the mean \pm standard error. Comparisons between clinicopathological characteristics and expression ratios $(\mathrm{T} / \mathrm{N})$ of LHX2 and miR-124 in NSCLC tissues were performed using nonparametric tests (Mann-Whitney U test for 2 groups, Kruskal-Wallis test for $\geq 3$ groups). For cell lines, differences
Table II. Comparison of various clinicopathological parameters with LHX2 mRNA and miR-124 expression in 40 NSCLC samples.

\begin{tabular}{|c|c|c|c|}
\hline \multirow[b]{2}{*}{ Parameter } & \multirow[b]{2}{*}{$\mathrm{n}$} & \multicolumn{2}{|c|}{ Ratio of expression $(\mathrm{T} / \mathrm{N})$} \\
\hline & & LHX2 mRNA & miR-124 \\
\hline \multicolumn{4}{|l|}{ Age } \\
\hline$\leq 65$ & 18 & $20.61 \pm 7.69$ & $0.73 \pm 0.19$ \\
\hline$>65$ & 22 & $7.41 \pm 2.55$ & $0.68 \pm 0.22$ \\
\hline${ }^{\text {aP-value }}$ & & 0.086 & 0.864 \\
\hline \multicolumn{4}{|l|}{ Sex } \\
\hline Male & 28 & $13.56 \pm 4.96$ & $0.67 \pm 0.20$ \\
\hline Female & 12 & $12.80 \pm 5.67$ & $6.03 \pm 5.23$ \\
\hline${ }^{\text {aP-value }}$ & & 0.929 & 0.144 \\
\hline \multicolumn{4}{|l|}{ Smoking status } \\
\hline No & 20 & $12.13 \pm 3.91$ & $3.82 \pm 3.15$ \\
\hline Yes & 20 & $14.54 \pm 6.68$ & $0.73 \pm 0.27$ \\
\hline${ }^{\text {aP }}$-value & & 0.892 & 0.298 \\
\hline \multicolumn{4}{|c|}{ Lymph node metastasis } \\
\hline No & 20 & $14.13 \pm 6.67$ & $3.76 \pm 3.15$ \\
\hline Yes & 20 & $12.54 \pm 3.95$ & $0.79 \pm 0.27$ \\
\hline${ }^{\text {aP-value }}$ & & 0.267 & 0.543 \\
\hline \multicolumn{4}{|l|}{ Histology } \\
\hline Adenocarcinoma & 23 & $10.16 \pm 3.71$ & $3.38 \pm 2.74$ \\
\hline $\begin{array}{l}\text { Squamous cell } \\
\text { carcinoma }\end{array}$ & 14 & $18.37 \pm 8.82$ & $0.89 \pm 0.37$ \\
\hline Others & 3 & $14.17 \pm 13.59$ & $0.29 \pm 0.12$ \\
\hline${ }^{\text {b}} \mathrm{P}$-value & & 0.205 & 0.669 \\
\hline
\end{tabular}

Data are presented as the mean \pm standard error. ${ }^{\text {a Mann-Whitney }}$

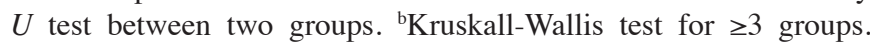
NSCLC, non-small cell lung cancer; T, NSCLC tissues; N, paired noncancerous lung tissues; miR, microRNA; LHX2, LIM-homeobox domain 2 .

between 2 groups were assessed using an unpaired t-test (two-tailed), and data are presented as the mean \pm standard deviation. Correlation between two groups was analyzed using the Pearson's correlation test. $\mathrm{P}<0.05$ was considered to indicate a statistically significant difference.

\section{Results}

LHX2 expression is increased in NSCLC cells and tissues. LHX2 is involved in various types of human cancer, including NSCLC (18). To further verify whether LHX2 expression is associated with NSCLC, the present study first examined the expression level of LHX2 in seven NSCLC cell lines. As shown in Fig. 1A and B, the levels of LHX2 mRNA and protein expression were significantly higher in seven NSCLC cell lines (H226, LTEP-a-2, A549, 95C, H460, 95D and H1299) compared with the control HBE cells. Subsequently, the level of LHX2 mRNA expression was detected in 40 paired NSCLC tissues and adjacent noncancerous lung tissues, and this analysis revealed that 


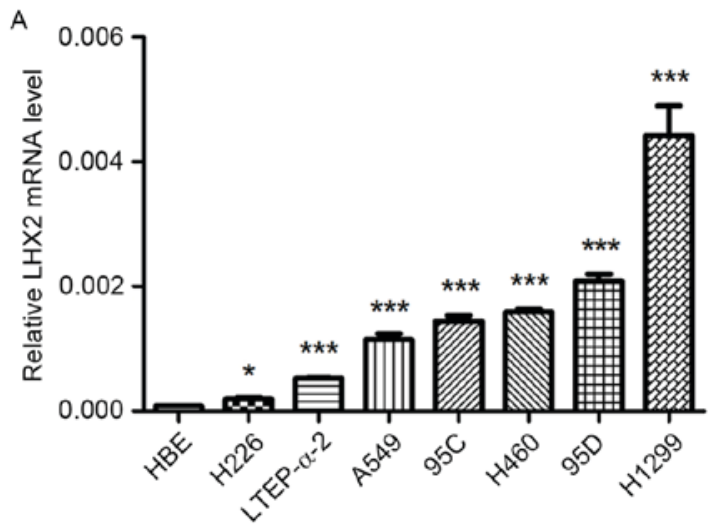

C



E

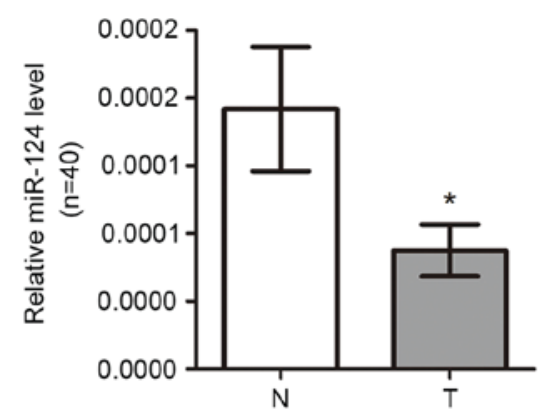

B

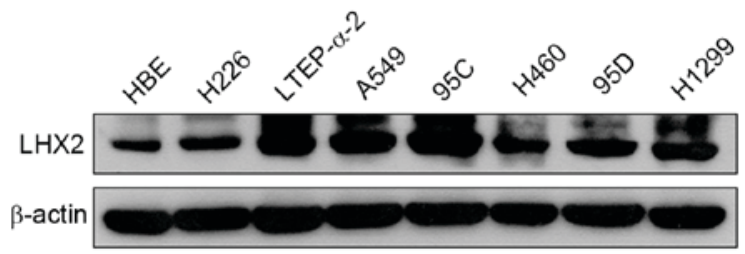

D

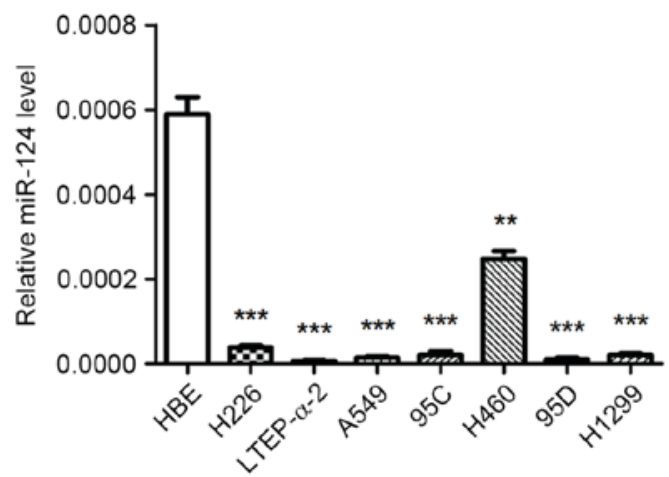

$\mathrm{F}$



Figure 1. High LHX2 and low miR-124 expression levels in human NSCLC cells and tissues. (A) RT-qPCR analysis of LHX2 mRNA levels in HBE and NSCLC cells. LHX2 mRNA expression levels are presented as a relative index normalized against $\beta$-actin. Data is presented as the mean \pm standard deviation of three replicates. (B) Western blot analysis of LHX2 protein levels in HBE and NSCLC cells. $\beta$-actin was used as the loading control. (C) Difference in relative LHX2 mRNA expression levels between 40 paired tumor tissues and adjacent noncancerous tissues. Data were analyzed using the paired t-test and presented as the mean \pm standard error. (D) RT-qPCR analysis of miR-124 expression levels in HBE and NSCLC cells. miR-124 expression levels were expressed as a relative index normalized against U6. (E) Relative miR-124 expression levels in 40 paired tumor tissues and adjacent noncancerous tissues. Difference in miR-124 expression level between tumor tissues and adjacent noncancerous tissues was analyzed by paired t-test. Data are presented as the mean \pm standard error. (F) Correlation between miR-124 and LHX2 mRNA expression levels in 40 paired NSCLC tissues. $\mathrm{x}$ and y axes represent the $\log _{10}$ transformed fold change of tumor/noncancerous tissue mRNA expression ratios of miR-124 and LHX2, respectively. ${ }^{*} \mathrm{P}<0.05 ;{ }^{* *} \mathrm{P}<0.01 ;{ }^{* * * *} \mathrm{P}<0.001$. NSCLC, non-small cell lung cancer; RT-qPCR, reverse transcription-quantitative polymerase chain reaction; LHX2, LIM-homeobox domain 2; miR, microRNA; HBE, human bronchial epithelial; $\mathrm{T}$, tumor; $\mathrm{N}$, noncancerous lung tissues.

LHX2 mRNA expression was significantly higher in NSCLC tissues compared with the paired noncancerous lung tissues (Fig. 1C). When classified by various clinicopathological characteristics, NSCLC samples did not reveal any difference in the LHX2 mRNA expression level ratio (T/N) (Table II).

Expression of miR-124 is decreased and negatively associated with the level of LHX2 expression in NSCLC cells and tissues. As presented in Fig. 1D, the expression of miR-124 was significantly lower in seven NSCLC cell lines (H226, LTEP-a-2, A549, 95C, H460, 95D and H1299) compared with the expression in HBE cells. Furthermore, miR-124 expression level was significantly lower in NSCLC tissues compared with the paired noncancerous lung tissues (Fig. 1E). No significant difference in miR-124expression ratio $(\mathrm{T} / \mathrm{N})$ was revealed between NSCLC samples when classified by various clinicopathological characteristics (Table II). Of note, the ratio of miR-124 expression level (T/N) was inversely associated 

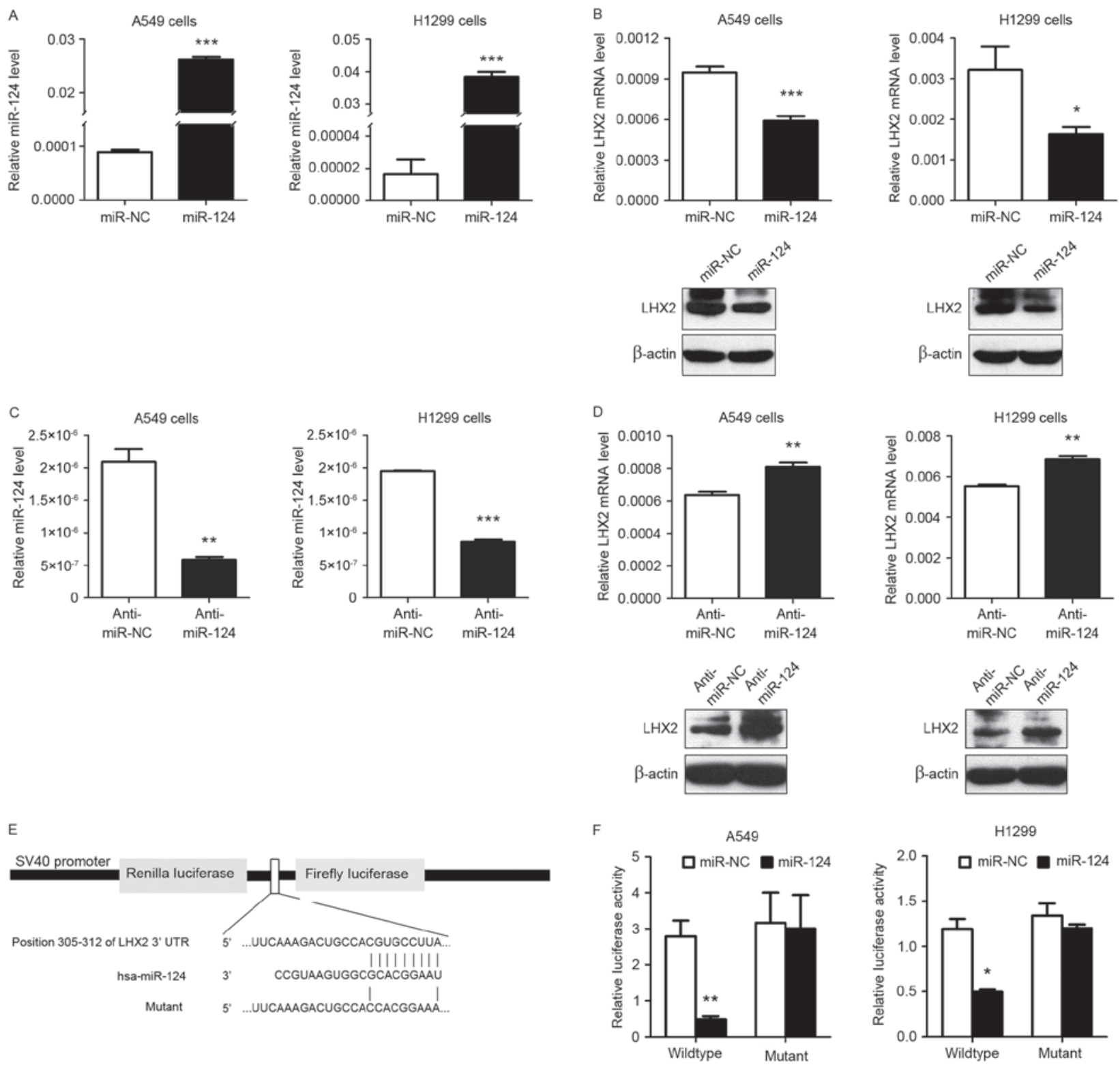

Figure 2. miR-124 reduces LHX2 expression level in NSCLC cells by targeting LHX2 3'-UTR. (A) RT-qPCR analysis of miR-124 expression levels in A549 and H1299 cells transfected with miR-124 mimics or miR-NC for $48 \mathrm{~h}$. Scrambled sequences were used as miR-NC, and U6 was used as an internal control. (B) LHX2 mRNA and protein expression levels in A549 and H1299 cells transfected with miR-124 mimics or miR-NC for $48 \mathrm{~h}$. (C) miR-124 expression levels in A549 and H1299 cells transfected with miR-124 inhibitor (anti-miR-124) or negative control (anti-miR-NC). (D) LHX2 mRNA and protein expression levels in A549 and H1299 cells transfected with anti-miR-124 or anti-miR-NC. (E) Schematic diagram showing the cloning of one predicted miR-124 binding site of LHX2 3'-UTR in psiCHECK-2 luciferase constructs. (F) Relative luciferase activities of the wild-type or mutant LHX2 3'-UTR reporter gene in A549 and H1299 cells transfected with miR-124 mimics or miR-NC. Relative Renilla luciferase activity was determined by normalization to firefly luciferase activity. Data is presented as the mean \pm standard deviation of three replicates. ${ }^{\prime} \mathrm{P}<0.05 ;{ }^{* *} \mathrm{P}<0.01 ;{ }^{* * *} \mathrm{P}<0.001 .3^{\prime}-\mathrm{UTR}, 3^{\prime}$ untranslated region; miR, microRNA; LHX2, LIM-homeobox domain 2; NSCLC, non-small cell lung cancer; RT-qPCR, reverse transcription-quantitative polymerase chain reaction; NC, negative control.

with LHX2 mRNA expression level (T/N) in 40 paired tissues (Fig. 1F). The results, analyzed using Pearson's correlation test, suggested that there may be a correlation between miR-124 and LHX2 expression levels in NSCLCs.

miR-124 suppresses LHX2 expression level by targeting LHX2 3'-UTR in NSCLC cells. As shown in Fig. 2A, a markedly increased expression level of miR-124 was observed in A549 and H1299 cells when transiently transfected with miR-124 mimic. Notably, miR-124 overexpression significantly inhibited LHX2 mRNA and protein expression levels in A549 and H1299 cells (Fig. 2B), and miR-124 downregulation (Fig. 2C) markedly promoted LHX2 mRNA and protein expression levels in A549 and H1299 cells (Fig. 2D), suggesting that miR-124 served an important role in downregulating LHX2 in NSCLC. To further investigate the molecular mechanisms underlying this inhibition effect, the present study first used TargetScanHuman (version 7.0) software, which identified LHX2 3'-UTR to be a putative target of miR-124. Therefore, in order to confirm this, the present study subcloned LHX2 3'-UTR containing the miR-124 binding site (wild-type/mutant) into psiCHECK-2 vectors (Fig. 2E) and transiently co-transfected the reporter vector with miR-124 mimic into NSCLC cell lines A549 and H1299. The results of the luciferase reporter assay demonstrated that miR-124 

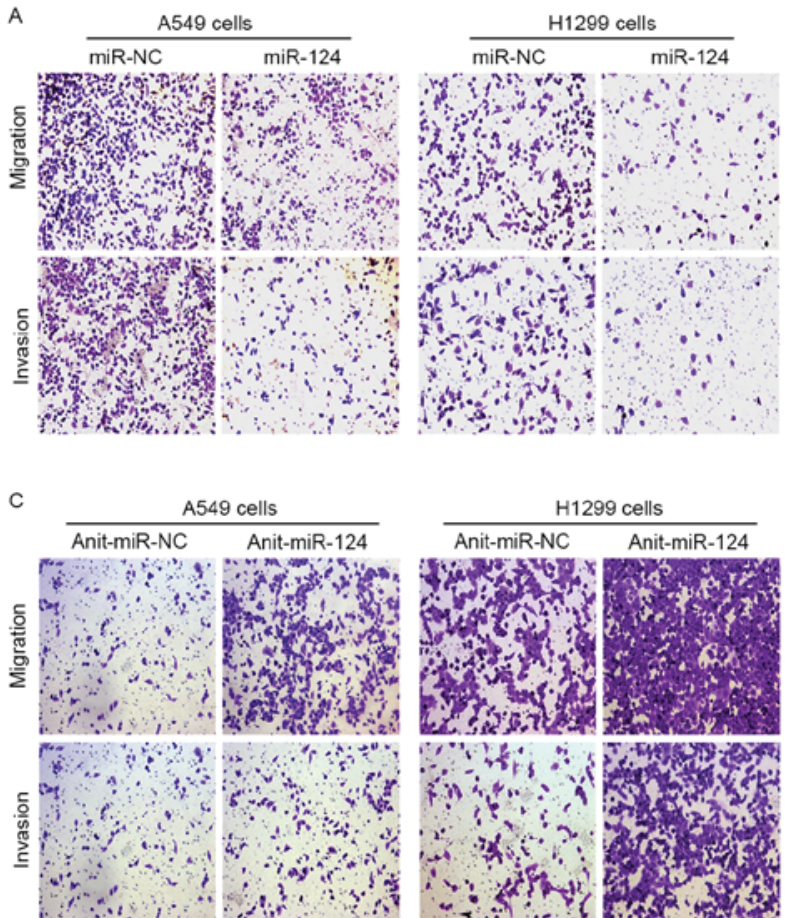
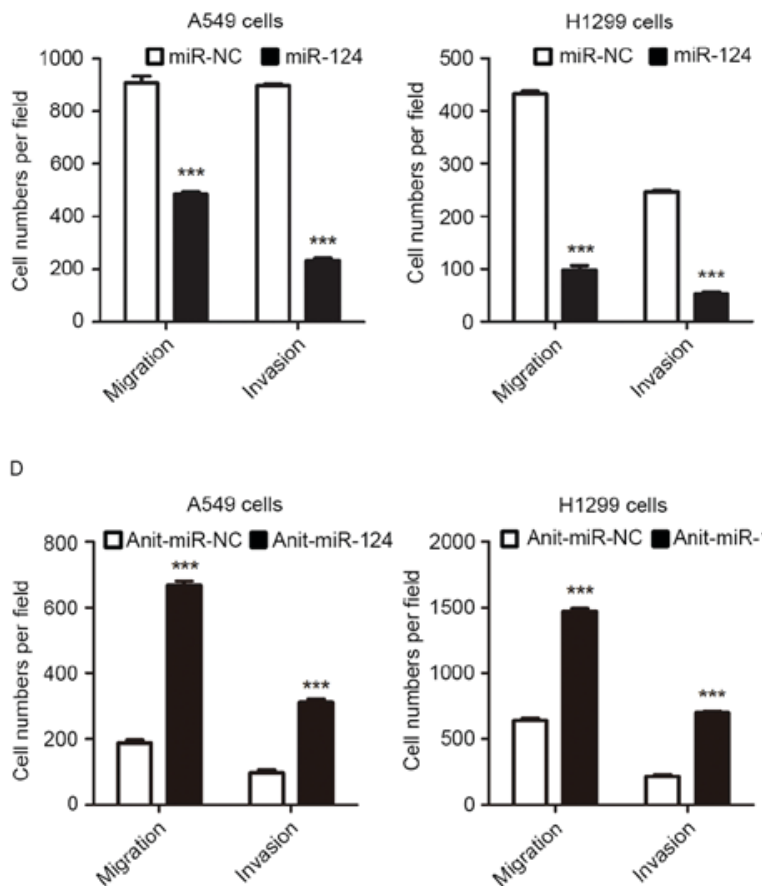

Figure 3. miR-124 suppresses migration and invasion of NSCLC cells. (A) Transwell assays of A549 cells and H1299 cells transfected with miR-124 or miR-NC. Following transfection with miR-124 or miR-NC for $24 \mathrm{~h}$, A549 and H1299 cells were allowed to invade through an $8 \mu \mathrm{M}$ pore in Transwell chambers. Migrated and invaded cells were stained and counted in $\geq 3$ microscopic fields (magnification, $x 100$ ). (B) Bar charts presenting the number of miR-124 mimics or miR-NC-transfected cells that have undergone migration or invasion. Data are presented as the mean \pm standard deviation of three independent fields. (C) Transwell assays for A549 cells and H1299 cells transfected with anti-miR-124 or anti-miR-NC. (D) Bar charts presenting the number of anti-miR-124 or anti-miR-NC-transfected cells that have undergone migration or invasion. ${ }^{* * *} \mathrm{P}<0.001$. miR, microRNA; NSCLC, non-small cell lung cancer; $\mathrm{NC}$, negative control.

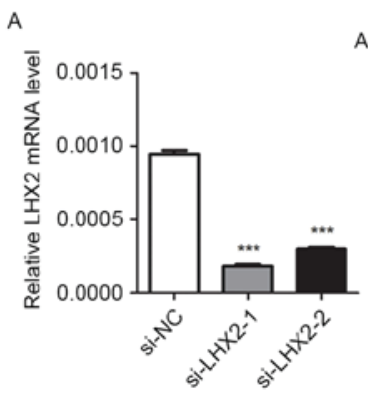

A549

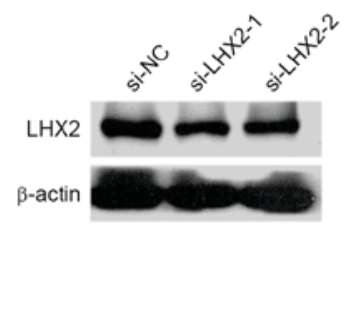

A549
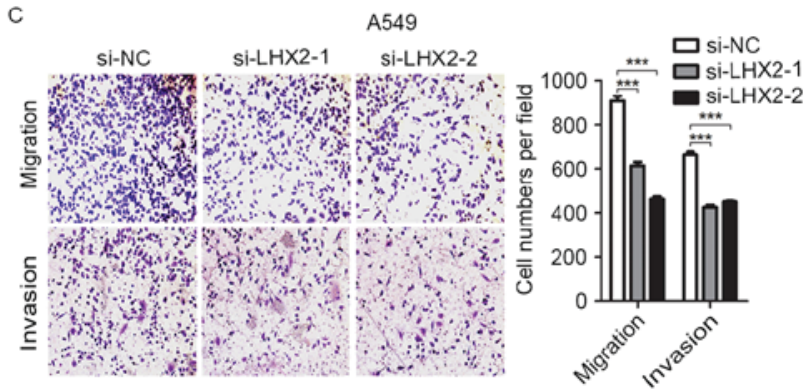

B

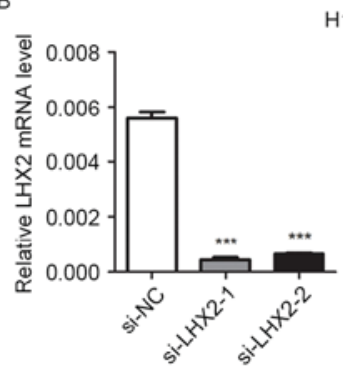

H1299

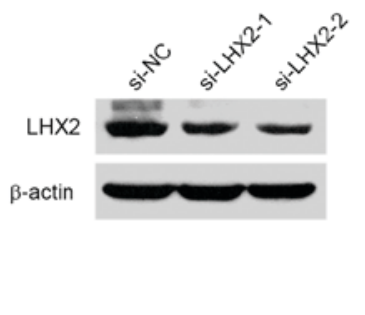

D

H1299
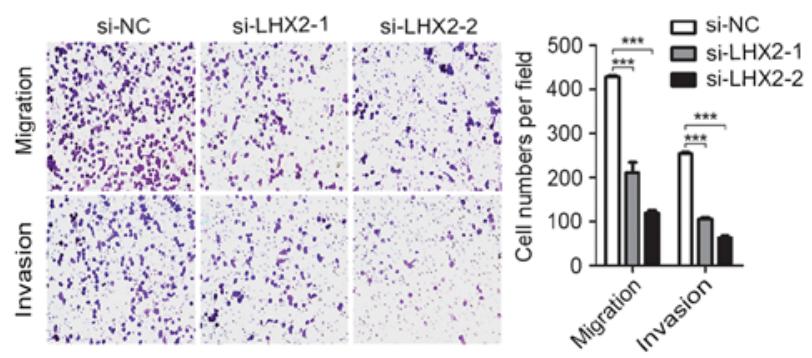

Figure 4. Knockdown of LHX2 inhibits migration and invasion of NSCLC cells. The levels of LHX2 mRNA and protein in (A) A549 cells and (B) H1299 cells transfected with si-LHX2-1, si-LHX2-2or si-NCfor 48 h. Transwell assays for (C) A549 and (D) H1299 cells transfected with si-LHX2-1, si-LHX2-2 or si-NC. LHX2-silenced A549 and H1299 cells were allowed to migrate and invade through an $8 \mu$ M pore in Transwell chambers. Migrated and invaded cells were stained and counted in $\geq 3$ microscopic fields (magnification, $x 100$ ). Data in the bar charts are presented as the mean \pm standard deviation of three independent fields. ${ }^{* * *} \mathrm{P}<0.001$. si, short interfering; LHX2, LIM-homeobox domain 2; NSCLC, non-small cell lung cancer; NC negative control.

significantly inhibited the luciferase activities in NSCLC cells transfected with the LHX2 3'-UTR wild-type plasmids, whereas
miR-124 did not suppress the luciferase activities in cells transfected with the mutant vectors (Fig. 2F). Taken together, the 
results indicated that miR-124 may directly target the 3'-UTR of LHX2 and thereby reduced the expression level of LHX2.

miR-124 overexpression or knockdown of LHX2 represses NSCLC cell migration and invasion. Given the observation that miR-124 may attenuate NSCLC metastasis by targeting MYO10 (13) and the results of the present study, whether repression of LHX2 expression by miR-124 may inhibit NSCLC cell migration and invasion was subsequently investigated. In support of this hypothesis, the Transwell assay revealed that A549 and H1299 cells overexpressing miR-124 exhibited an impaired ability to migrate and invade (Fig. 3A and B). Furthermore, A549 and H1299 cells with downregulated miR-124 expression demonstrated increased migratory and invasive abilities (Fig. 3C and D). Notably, knockdown of LHX2 (Fig. 4A and B) suppressed migratory and invasive abilities of A549 and H1299 cells (Fig. 4C and D). Taken together, the results suggested that miR-124 may attenuate NSCLC cell migration and invasion by targeting LHX2.

\section{Discussion}

LHX2 serves important roles in multiple biological processes, including embryo development (16), cell fate decision, proliferation (21) and cell differentiation (22). Therefore, aberrant expression of LHX2 may be associated with certain human diseases, including cancer. High levels of LHX2 were expressed in pancreatic ductal adenocarcinoma (23). In present study, it was revealed that LHX2 was highly expressed in NSCLC cells and tissues. Although the authors of the present study have previously reported that knockdown of LHX2 attenuated NSCLC cell proliferation (18), whether LHX2 expression affects NSCLC cell migration and invasion has not yet been investigated. Kuzmanov et al (17) reported that LHX2 functioned as a promoter of metastasis in breast cancer cells. In the present study, the findings indicated that knockdown of LHX2 significantly inhibited the aggressive abilities of NSCLC cells.

Subsequently, the present study investigated the miRNA-mediated mechanism underlying LHX2 regulation in NSCLC cell migration and invasion. Considering the idea that miRNAs serve a key role in regulating various gene expression levels at the post-transcriptional level (24) and in silico prediction which identified the 3'-UTR of LHX2 transcript to be a putative target of miR-124, the present study performed cell-based and biochemical analyses to confirm this. Firstly, the present study revealed that LHX2 expression level was markedly downregulated and upregulated in NSCLC cells when transiently transfected with miR-124 mimic and miR-124 inhibitor, respectively. Secondly, luciferase reporter assays demonstrated that miR-124 repressed LHX2 expression level by targeting a specific site of the LHX2 3'-UTR. To the best of our knowledge, this is the first evidence that miR-124 targets LHX2 and inhibits its expression in NSCLC cells. In addition, the present study observed that miR-124 overexpression inhibited NSCLC cell migration and invasion, which was consistent with the results from a previous study by Sun et al (13). The phenotype of miR-124 overexpression was the same as the phenotype of NSCLC cells with knockdown of LHX2, which presented an attenuated aggressive ability, suggesting that miR-124 may inhibit NSCLC cell migration and invasion by targeting LHX2. Of note, miR-124 may also target MYO10 and inhibit NSCLC metastasis (13). This is not surprising because single miRNA have been reported to regulate various target genes to exert its function (25). In further support of this, miR-124 directly targets ESX/epidermal growth factor receptor or talin 1 to suppress cell invasion in head and neck squamous cell carcinoma (11) and prostate cancer (26).

Recently, a low level of miR-124 expression was demonstrated to be significantly associated with positive lymph node metastasis and poor prognosis in human cancer, including NSCLC $(14,15)$. The present study observed that miR-124 inhibition increased the migratory and invasive abilities of NSCLC cells, and miR-124 was downregulated in NSCLC tissues. However, due to the limited sample size, the present study failed to reveal the significant association of miR-124 expression level with lymph node metastasis in NSCLC tissues. Furthermore, the results demonstrated that a low expression level of miR-124 was inversely associated with a high level of LHX2 expression in NSCLCs.

In conclusion, the present study provided the first evidence that LHX2 is involved in NSCLC cell migration and invasion, which was regulated at least partially by miR-124.Mechanistically, miR-124 reduced LHX2 expression by directly targeting the LHX2 3'-UTR. The results of the present study suggest that overexpression of miR-124 or silencing of LHX2 may provide a therapeutic strategy for advanced NSCLC.

\section{Acknowledgements}

The present study was supported in part by grants from the National Natural Science Foundation of China (grant nos. 81372277, 81171894 and81502498), the Jiangsu Province's Key Provincial Talents Program (grant no. RC2011106), the Science and Technology Committee of Jiangsu Province (grant no. BK20131159), the '333' Project of Jiangsu Province Government (grant no. 2011-III-2166), the Graduate Innovation Project of Jiangsu Province (grant no. CXZZ13_0830), the Natural Science Foundation of the Jiangsu Higher Education Institution (grant no. 14KJB0017), the Soochow Scholar Project of Soochow University (grant no. SSPSU2010-51) and a project funded by the Priority Academic Program Development of Jiangsu Higher Education Institution (PAPD-XL2014014).

\section{References}

1. Herbst RS, Heymach JV and Lippman SM: Lung cancer. N Engl J Med 359: 1367-1380, 2008.

2. DeSantis CE, Lin CC, Mariotto AB, Siegel RL, Stein KD, Kramer JL, Alteri R, Robbins AS and Jemal A: Cancer treatment and survivorship statistics, 2014. CA Cancer J Clin 64: 252-271, 2014.

3. Liu RY, Zeng Y, Lei Z, Wang L, Yang H, Liu Z, Zhao J and Zhang HT: JAK/STAT3 signaling is required for TGF- $\beta$-induced epithelial-mesenchymal transition in lung cancer cells. Int $\mathrm{J}$ Oncol 44: 1643-1651, 2014.

4. Guo H, Ingolia NT, Weissman JS and Bartel DP: Mammalian microRNAs predominantly act to decrease target mRNA levels. Nature 466: 835-840, 2010.

5. Wilson RC and Doudna JA: Molecular mechanisms of RNA interference. Annu Rev Biophys 42: 217-239, 2013.

6. Butz H, Racz K, Hunyady L and Patocs A: Crosstalk between TGF- $\beta$ signaling and the microRNA machinery. Trends Pharmacol Sci 33: 382-393, 2012. 
7. Lei Z, Xu G, Wang L, Yang H, Liu X, Zhao J and Zhang HT: MiR-142-3p represses TGF- $\beta$-induced growth inhibition through repression of TGF $\beta R 1$ in non-small cell lung cancer. FASEB J 28: 2696-2704, 2014.

8. Arabkheradmand A, Safari A, Seifoleslami M, Yahaghi E and Gity M: Down-regulated microRNA-124 expression as predictive biomarker and its prognostic significance with clinicopathological features in breast cancer patients. Diagn Pathol 10: 178, 2015

9. Ibarrola-Villava M, Llorca-Cardeñosa MJ, Tarazona N, Mongort C, Fleitas T, Perez-Fidalgo JA, Roselló S, Navarro S, Ribas G and Cervantes A: Deregulation of ARID1A, CDH1, cMET and PIK3CA and target-related microRNA expression in gastric cancer. Oncotarget 6: 26935-26945, 2015

10. Wang X, Wu Q, Xu B, Wang P, Fan W, Cai Y, Gu X and Meng F: miR-124 exerts tumor suppressive functions on the cell proliferation, motility and angiogenesis of bladder cancer by fine-tuning UHRF1. FEBS J 282: 4376-4388, 2015.

11. Zhang M, Piao L, Datta J, Lang JC, Xie X, Teknos TN, Mapp AK and Pan Q: miR-124 regulates the epithelial-restricted with serine box/epidermal growth factor receptor signaling axis in head and neck squamous cell carcinoma. Mol Cancer Ther 14 2313-2320, 2015

12. Xi ZW, Xin SY, Zhou LQ, Yuan HX, Wang Q and Chen KX: Downregulation of rho-associated protein kinase 1 by miR-124 in colorectal cancer. World J Gastroenterol 21: 5454-5464, 2015.

13. Sun Y, Ai X, Shen S and Lu S: NF- $\kappa$ B-mediated miR-124 suppresses metastasis of non-small-cell lung cancer by targeting MYO10. Oncotarget 6: 8244-8254, 2015.

14. Dong LL, Chen LM, Wang WM and Zhang LM: Decreased expression of microRNA-124 is an independent unfavorable prognostic factor for patients with breast cancer. Diagn Pathol 10: 45,2015 .

15. Zhang Y, Li H, Han J and Zhang Y: Down-regulation of microRNA-124 is correlated with tumor metastasis and poor prognosis in patients with lung cancer. Int J Clin Exp Pathol 8: 1967-1972, 2015
16. Porter FD, Drago J, Xu Y, Cheema SS, Wassif C, Huang SP, Lee E, Grinberg A, Massalas JS, Bodine D, et al: Lhx2, a LIM homeobox gene, is required for eye, forebrain, and definitive erythrocyte development. Development 124: 2935-2944, 1997.

17. Kuzmanov A, Hopfer U, Marti P, Meyer-Schaller N, Yilmaz M and Christofori G: LIM-homeobox gene 2 promotes tumor growth and metastasis by inducing autocrine and paracrine PDGF-B signaling. Mol Oncol 8: 401-416, 2014

18. Shi X, Zhan L, Xiao C, Lei Z, Yang H, Wang L, Zhao J and Zhang HT: miR-1238 inhibits cell proliferation by targeting LHX2 in non-small cell lung cancer. Oncotarget 6: 19043-19054, 2015.

19. Mountain CF: Revisions in the international system for staging lung cancer. Chest 111: 1710-1717, 1997.

20. Livak KJ and Schmittgen TD: Analysis of relative gene expression data using real-time quantitative PCR and the 2(-Delta Delta C(T)) Method. Methods 25: 402-408, 2001.

21. Pinto do OP, Kolterud A and Carlsson L: Expression of the LIM-homeobox gene LH2 generates immortalized steel factor-dependent multipotent hematopoietic precursors. EMBO J 17: 5744-5756, 1998

22. Chou SJ and O'Leary DD: Role for Lhx2 in corticogenesis through regulation of progenitor differentiation. Mol Cell Neurosci 56: 1-9, 2013.

23. Zhou F, Gou S, Xiong J, Wu H, Wang C and Liu T: Oncogenicity of LHX2 in pancreatic ductal adenocarcinoma. Mol Biol Rep 41: 8163-8167, 2014.

24. Ohtsuka M, Ling H, Doki Y, Mori M and Calin GA: MicroRNA processing and human cancer. J Clin Med 4: 1651-1667, 2015

25. Lim LP, Lau NC, Garrett-Engele P, Grimson A, Schelter JM, Castle J, Bartel DP, Linsley PS and Johnson JM: Microarray analysis shows that some microRNAs downregulate large numbers of target mRNAs. Nature 433: 769-773, 2005.

26. Zhang W, Mao YQ, Wang H, Yin WJ, Zhu SX and Wang WC: MiR-124 suppresses cell motility and adhesion by targeting talin 1 in prostate cancer cells. Cancer Cell Int 15: 49, 2015. 